\title{
Scientometric analyses and visualization of scientific outcome on Nipah virus
}

\author{
Nirmal Singh*, R. S. Brar, Shankar B. Chavan and Jaswant Singh
}

This study presents scientometric analysis and network visualization of journal articles on Nipah virus for a better understanding of research trends on the subject. Metadata of journal articles were retrieved from Scopus database in 2018. After filtration of irrelevant and incomplete records, 1007 records were analysed to examine the publishing trends on Nipah virus. The network visualization was accomplished using 'VOSviewer', 'Gephi' and 'Sciencescape' software. Results revealed inconsistent growth of articles on the subject. There were only few productive authors with $\geq 15$ records, but others contributed on the subject occasionally. The United States (as a country) and North America (as continent) led the world in terms of contribution to the number of articles ( $n=469$ and 522 records respectively). Six leading institutes contributed to $\geq 50$ records each. Out of 373 journals, ten core journals published $31 \%$ of the total journal articles. Predominant topics discussed in the articles included: characterization, pathogenesis, clinical symptoms, transmission, outbreaks and vaccine development. A great degree of inter-country and inter-continent collaboration was detected among the US, Australia, Malaysia, United Kingdom and Bangladesh, depicting that the developed world is working in association with the developing nations to tackle the problem.

Keywords: Bibliometrics, collaboration, Nipah virus, scientometrics, visualization.

\section{Background}

The Nipah virus (NiV) is a new addition to the growing list of emerging zoonotic diseases. Since its identification, NiV infection has attracted scientific attention due to high morbidity and mortality rates in both humans and animals ${ }^{1}$. Recently, a World Health Organization (WHO) report included Nipah diseases under the 'list of blueprint priority diseases' requiring an urgent need for accelerated research and development ${ }^{2}$.

The virus was first isolated from the cerebrospinal fluid of a human patient from Nipah village in Malaysia during an outbreak of viral encephalitis in 1998-99 (refs 3-5). NiV first appeared in domestic pigs in Malaysia in 1998 and spread to nearby Singapore in 1999 (refs 6 and 7). Fruit bats of the Pteropodidae family are the natural host of the virus ${ }^{8}$. NiV infection has been identified among a number of domestic and companion animals, including dog, cat, goat, horse and sheep ${ }^{9}$. Human-to-

\footnotetext{
Nirmal Singh and R. S. Brar are in the Guru Angad Dev Veterinary and Animal Sciences University, Ludhiana 141 004, India; Shankar B. Chavan is in the Indian Institute of Technology Delhi, New Delhi 110 016, India and Jaswant Singh is in the University of Saskatchewan, Saskatoon, Canada.

For correspondence. (e-mail: nirma102@yahoo.co.in)
}

human transmission of infection has been reported, raising concerns for health workers. Virus outbreaks affecting the human population occurred in Bangladesh and India in 2003, 2004, 2007, 2008 and 2018, without concurrent domestic animal outbreaks ${ }^{10}$. Between 2001 and May 2018, 261 cases of NiV were reported in Bangladesh and 85 in India that have taken 198 and 62 lives in these countries respectively. Thus continuous research efforts are essential to counter this life-threatening virus and the literature on the subject is central for further research and development. A scientometric study on Nipah virus can (i) provide an outline of the literary outcome on the subject, (ii) be useful to collect and organize information resources and (iii) strengthen research collaborations. Further analysis of collaboration networks can facilitate the visualization of scientific knowledge flows. A few scientometric studies have been conducted on Nipah virus; however there are no studies of collaboration trends and network visualization. Safahieh et al. ${ }^{11}$ analysed the scientometric variables of 462 articles on Nipah virus published during 1999-2010. Gupta et al. ${ }^{12}$ conducted a scientometric study on publications during 1999-2018 on Nipah virus. The authors reported prolific authors, institutions and countries in terms of the respective number of publications. However, inter-author collaborations, interinstitution collaborations and international collaborations 
remained out of the scope of the study. Gupta et al. ${ }^{12}$ also reported the subject-wise distribution of publications as given under subject categories depicted in Scopus. Though the authors enlisted the most frequently used keywords, they neither reported their co-occurrences nor performed subject analyses based on such co-occurrences.

The emphasis on authorship, institutional and geographical collaborations, institutional-journal publishing trends and subject analyses based on co-occurrence of keywords applying network visualization differentiates the present study from the previous ones. The collaboration analyses using network visualization have also been presented.

This study is aimed to achieve the following objectives: (i) Examine the scientific productivity on $\mathrm{NiV}$ based on research articles cited in Scopus database. (ii) Identify the most productive authors, institutions and countries and develop collaboration visualization maps. (iii) Determine the core journals publishing articles on $\mathrm{NiV}$ and analyse the institutional-journal publishing trends. (iv) Develop subject map based on author keywords to present an overview of the current research trends.

\section{Methodology}

\section{Dataset}

For the purpose of this study, data were accessed from the Scopus database on 31 May 2018 using the phrases 'nipah' and 'nipah virus'. The earliest retrieved record was from the year 1999. The Boolean operator 'OR' was applied to obtain results due to either of the phrases. The search was filtered for occurrence of phrase terms in the title, abstract and keywords of records. The string search retrieved 1116 entries. The data were downloaded in tabdelimited text (CSV) format. Initially, an attempt was made to analyse all records using network visualization software. Since journal articles and other types of publication (e.g. book chapters) have unsymmetrical bibliographical fields, problems were encountered in the visualization of data. Thus, we limited the scope of the study to scientometric analyses of publications included in the peer-reviewed journals (including but not limited to the original research articles, reviews, editorials, short surveys and letters) that had similar bibliographical fields. The book chapters, conference papers, books and conference reviews, articles and reviews without authorship details and other missing details were removed from the file and data from 1007 records were used for scientometric analyses.

\section{Analysis of subject terms and institutions}

The words/phrases listed in the 'keyword' field in the Scopus data (i.e. those provided by the original publica- tions; defined as author-generated keywords) were considered for subject analysis $(n=2300$ terms, including 1122 unique keywords having one or more occurrences in the records retrieved). For the records where authorgenerated keywords were not available ( $n=599$ articles), the keywords were generated from the title of the articles (title-generated keywords) by removing helping verbs and other general words. The term 'Nipah virus' was excluded from analyses as all articles pertained to this subject. The singular and plural terms representing the same subject were further standardized to avoid duplicity in the occurrence of terms in the subject visualization map. The subject analysis was performed using authorgenerated and title-generated keywords $(n=5828$, unique keywords $=2385$ ) after removing duplicity due to plural forms.

Another problem was identified when the basic analysis of institutional contributions revealed that the institutional names were not listed in uniform style. Instances were observed where the name of a given institution was listed differently in different articles; for example, some articles listed complete name of the institution, others enlisted abbreviated names and sometimes name of the department/college/laboratory was also attached to the institution name. This made the assessment of interinstitutional productivity and collaborations impossible. Hence, all institutional names were examined individually and manually standardized. The institutions having multiple campuses at different places within a country were treated as a single institution when examining interinstitutional collaborations.

\section{Definitions}

Collaborative authorship: Publications with two or more authors were considered the outcome of collaborative authorship.

Collaborating countries: When authors from two or more countries contributed to a given article, their respective countries were considered as collaborating countries.

Collaborating institutions: When authors from two or more institutions contributed to the same article in joint authorship, their respective institutions were considered as collaborating institutions.

Collaboration connection: It is a link(s) between collaborating authors, collaborating institutions and collaborating countries (using VOSviewer and Gephi software).

Core journals: The journals that published 15 or more articles on $\mathrm{NiV}$ were considered as core journals.

\section{Data visualization}

The scientific productivity on Nipah virus was mapped using free-to-use software 'VOSviewer', 'Gephi' and 'Sciencescape'. The most productive authors and 


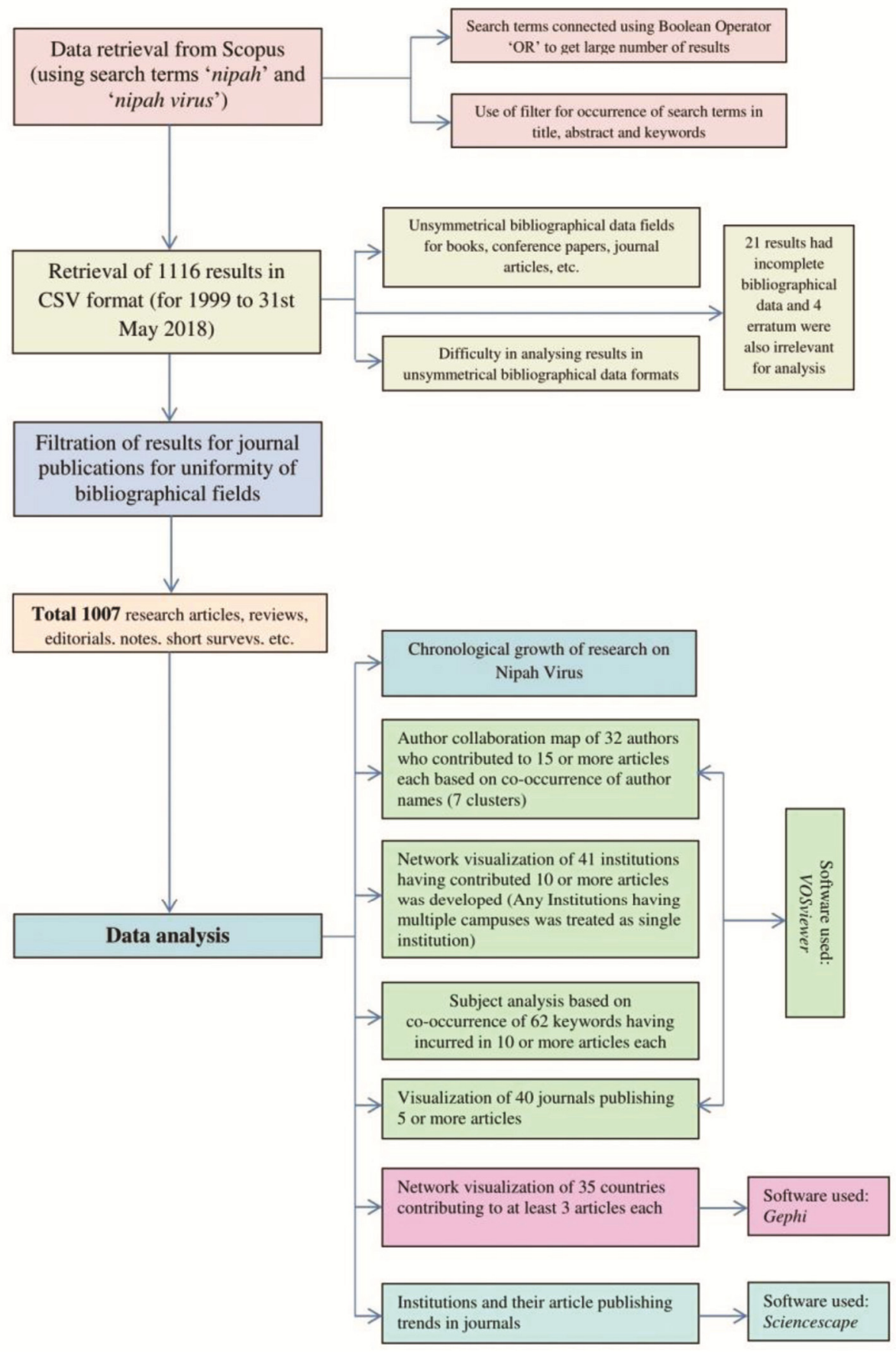

Figure 1. Flowchart of data collection and data analysis.

institutions were identified and collaborations among them were visualized using 'VOSviewer'. The clusters were developed using VOSviewer based on the cooccurrence of authors, institutions and subject terms. In VOSviewer, each cluster represents mutually related nodes and each node in a network falls under one cluster only ${ }^{13}$. The collaboration among countries was visualized through 'Gephi'. The network maps developed using 'VOSviewer' and 'Gephi' have nodes and edges. The edges are the links between nodes establishing the inter-connectivity of nodes representing authors, countries and subject terms. The thickness of edges represents the strength of collaboration between authors, countries and co-occurrence of keywords in subject analysis. The size of the nodes represents the number of articles contributed by the respective authors, countries and frequency of occurrence of keywords in the articles. The A-K-J Sankey feature of 'Sciencescape' was used to identify the institutional trends of publishing articles in journals. Figure 1 depicts a flowchart of various steps from data collection to data analysis. 


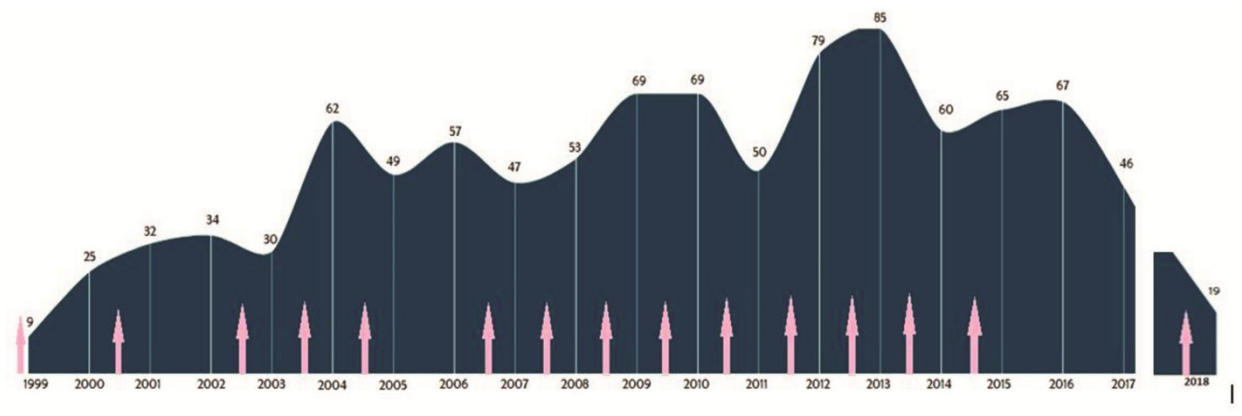

Figure 2. Chronological growth of publications.

\section{Results and discussion}

\section{Chronological trends in Nipah virus publications}

During 1999-2018, a total of 1116 publications were recorded in the Scopus database on Nipah virus, including 668 articles, 249 reviews, 45 book chapters, 34 conference papers, 36 editorials, 33 notes, 22 short surveys, 20 letters, four errata, three books and two conference reviews. The book chapters, conference papers, books and conference reviews were excluded from this study. In addition 21 articles and reviews without authorship and other details and four errata were also excluded. Finally, a total 1007 records, including 663 articles, 233 reviews, 36 editorials, 33 notes, 22 short surveys and 20 letters published in journals were analysed. Figure 2 shows the chronological growth of articles on Nipah virus published in the journals.

Data for the year 2018 were partial since they were retrieved at the end of May 2018. Arrows in Figure 2 indicate the years when outbreaks occurred.

The growth of publications on Nipah virus during the period under study was found to be uneven. The year of identification of Nipah virus, i.e. 1999 witnessed nine publications and yearly increase in the number of publications continued till 2002. After publication of 62 records in 2004, the following four years (2005-08) witnessed lesser number of publications. Maximum number of records were published in 2013. Thereafter, the number of publications fluctuated between 46 and 67 records (the year 2018 has been excluded as data were available partially).

\section{Most productive authors}

All except 19\% articles (191 single-author publications of 1007 records) have been an outcome of collaborative authorship. More than 42\% (428/1007) articles were contributed by $2-5$ authors and the $38 \%(388 / 1007)$ articles were authored by six or more co-authors each. In total, 2804 authors contributed to the 1007 records on Nipah virus. Thirty-five authors contributed to 15 or more articles each. Three of these 35 authors did not have any collaboration and were therefore excluded from the collaboration network analyses. There is little chance of these 32 authors having the same surname and first name. Therefore, the authorship collaboration map has been developed based on the data processed using VOSviewer without any further cross examination of author names (Figure 3). Five clusters have been formed using cluster schema of VOSviewer based on co-occurrences of author-terms in articles. The size of nodes represents the contribution of the respective authors in terms of number of articles and edges show the strength of links between authors. Three clusters have seven or more authors each, showing a strong collaborative research commitment at the national/international level.

In Figure 3, author names represent different clusters along with number of articles (D) and link strength (LS) of each author depicting their collaborations.

The clusters formed using cluster schema of the VOSviewer are given below:

Cluster 1: Bossart, K. N., Broder, C. C., Crameri, G., Dimitrov, D. S., Eaton, B. T., Feldmann, H., Marsh, G. A., Middleton, D., Rockx, B. and Wang, L. F.

Cluster 2: Bellini, W. J., Gurley, E. S., Hossain, M. J., Ksiazek, T. G., Lo, M. K., Luby, S. P., Rahman, M., Rolin, P. E. and Rota, P. A.

Cluster 3: Chua, K. B., Horvat, B., Moscona, A., Mungall, B. A., Porotto, M., Tan, C. T. and Wong, K. T. Cluster 4: Aguilar, H. C., Freiberg, A. N. and Lee, B. Cluster 5: Daszak, P., Epstein, J. H. and Field, H. E.

\section{Major contributing countries}

Authors from a total of 81 countries contributed to 1007 records. Five nations from the North America continent collectively contributed to the highest number of records $(n=525)$, followed by contribution of 392 records from 30 Asian nations and 289 records from 21 European countries. Australia/Oceania contributed to 182 records. Eighteen African countries added to 41 records and five South American nations contributed to 16 records. 


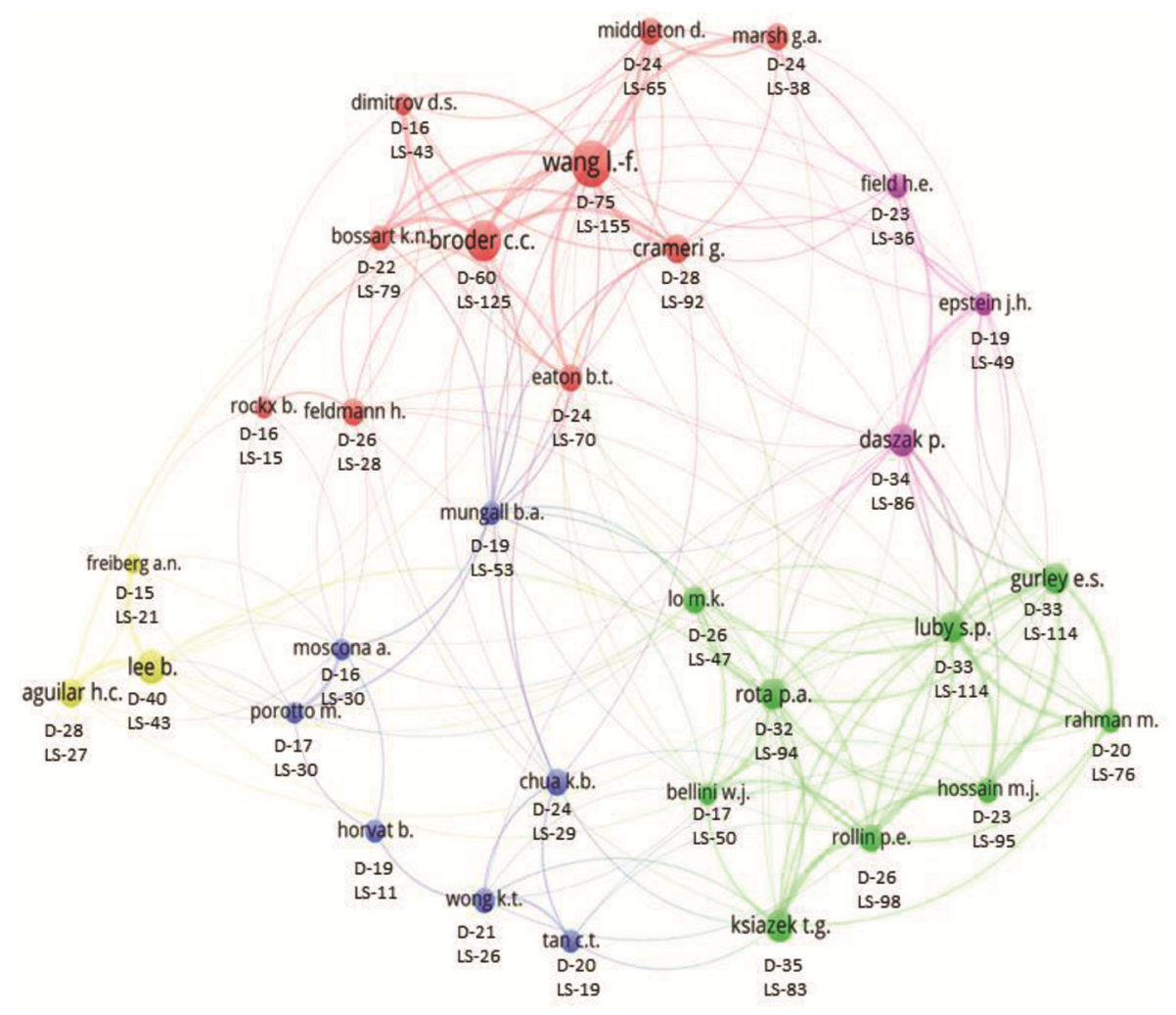

Figure 3. The most productive authors.

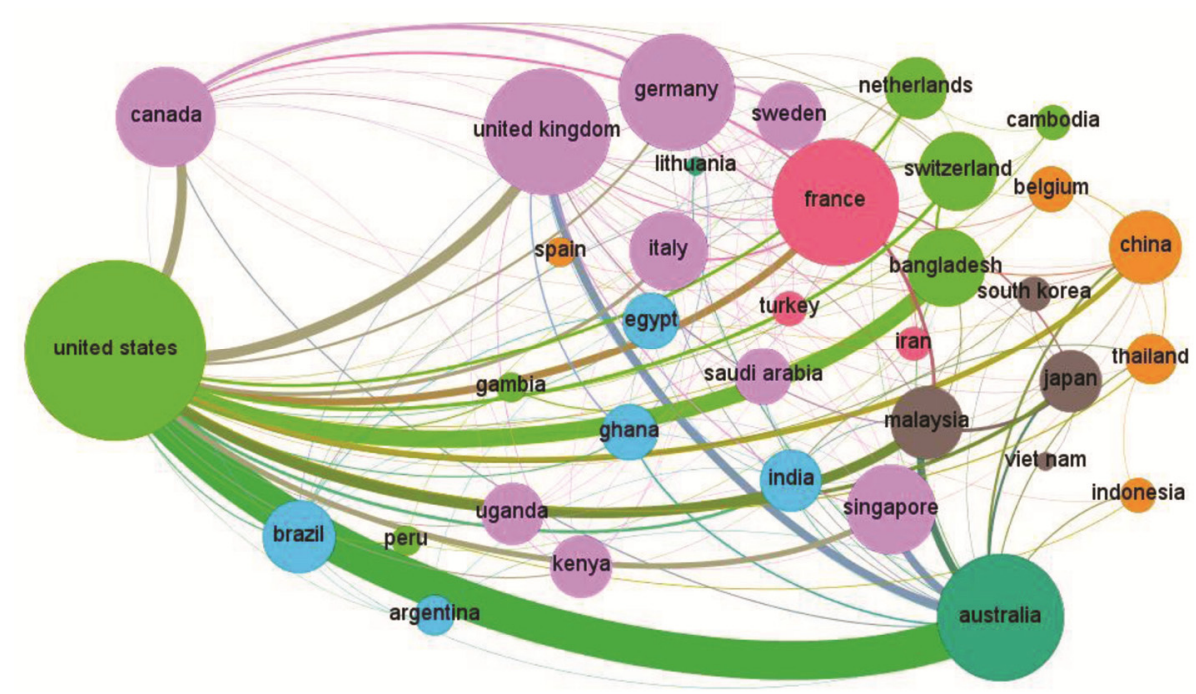

Figure 4. The most productive countries on Nipah virus.

Eight of these 81 countries did not have international collaboration. Eighteen countries from the African continent, 26 from Asia, 17 from Europe, 5 nations each from North America and South America, and two countries from Australia/Oceania worked in international collaboration on Nipah virus in the form of journal articles. The network visualization map of countries having contributed at least three articles on Nipah virus was developed using Gephi. Out of the resulting 37 countries, two (Greece and Poland) did not have any articles in international collaboration. Hence, these two nations were excluded while developing the international collaboration network. Figure 4 shows the top 35 countries with the highest number of articles on Nipah virus.

In Figure 4, D represents the number of articles, $\mathrm{L}$ the number of links, and LS the total link strength. The top 


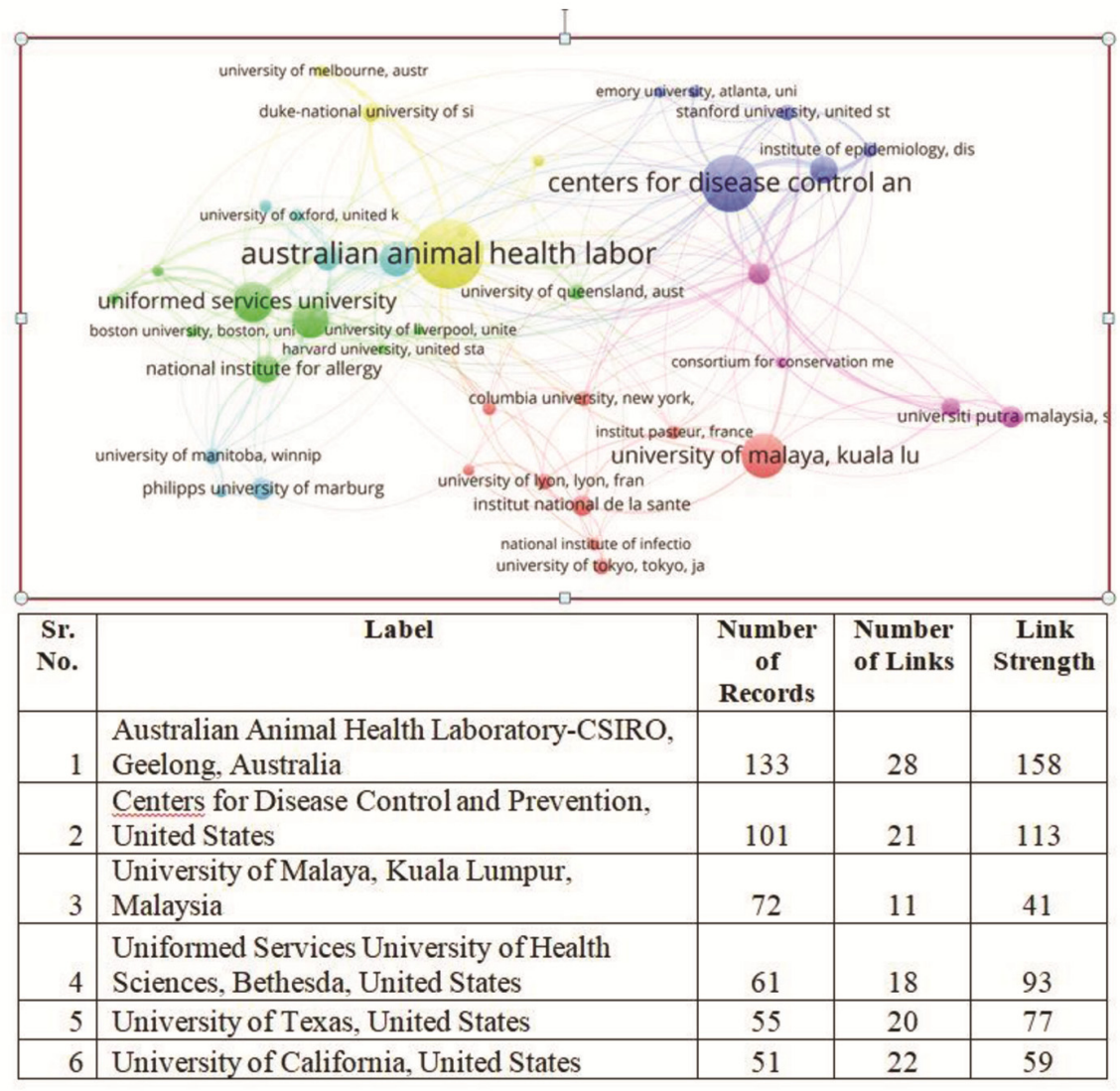

Figure 5. Collaboration map of the most productive institutions $(n=41)$ and scientometric details of institutions $(n=6)$ that contributed $\geq 50$ records.

10 countries in decreasing order of number of publications are: the United States: D-469, L-31, LS-318; Australia: D180, L-21, LS-181; Malaysia: D-125, L-11; LS-68; France: D-77, L-21, LS-69; United Kingdom: D-71, L-21, LS-78; Germany: D-54, L-19, LS-44; Canada: D-48, L-16, LS-55; Bangladesh: D-46, L-12, LS-58; India: D-45, L-9, LS-20 and Singapore: D-42, L-14, LS-45.

In Figure 4, there are seven country clusters. This visualization map has been developed using Gephi. Clusterwise countries are listed below:

Cluster 1: Canda, Germany, Italy, Kenya, Saudi Arabia, Singapore, Sweden, Uganda, United Kingdom.

Cluster 2: Bangladesh, Cambodia, Gambia, Netherlands, Peru, Switzerland, the United States.

Cluster 3: Argentina, Brazil, Egypt, Ghana, India.

Cluster 4: Belgium, China, Indonesia, Spain, Thailand.

Cluster 5: Japan, Malaysia, South Korea, Vietnam.

Cluster 6: Australia, Lithuania.

\section{Institutional productivity}

Authors from 846 institutions/organizations contributed to the 1007 records. Figure 5 shows the collaboration visualization map of 41 institutions (that contributed to $\geq 10$ records each). Scientometric data (number of articles, number of links, link strength) of the top 6 institutions ( $\geq 50$ records; individually or in collaboration) is also tabulated.

Seven clusters as given below were identified representing institutional collaborations:

Cluster 1: Australian Animal Health Laboratory-CSIRO, Geelong, Australia; Duke-National University of Singapore, Singapore; Memorial Sloan-Kettering Cancer Centre, New York, USA; National Cancer institute, National Institute of Health Sciences; Pennsylvania State University, USA; University of Cambridge, Cambridge, United Kingdom; Uniformed Services University of health Sciences; Uniformed Services University, Bethesda, USA and University of Melbourne, Australia.

Cluster 2: Centre National De La RechercheScientifique, France; Columbia University, New York, USA; Cornell University, New York, USA; Institut National De LA Sante Et De La Recherche; Institut Pasteur, France; National Institute of Infectious Diseases, Tokyo, Japan; University of Lyon, Lyon, France; University of Malaya and Kuala Lumpur, Malaysia; University of Tokyo, Japan. 


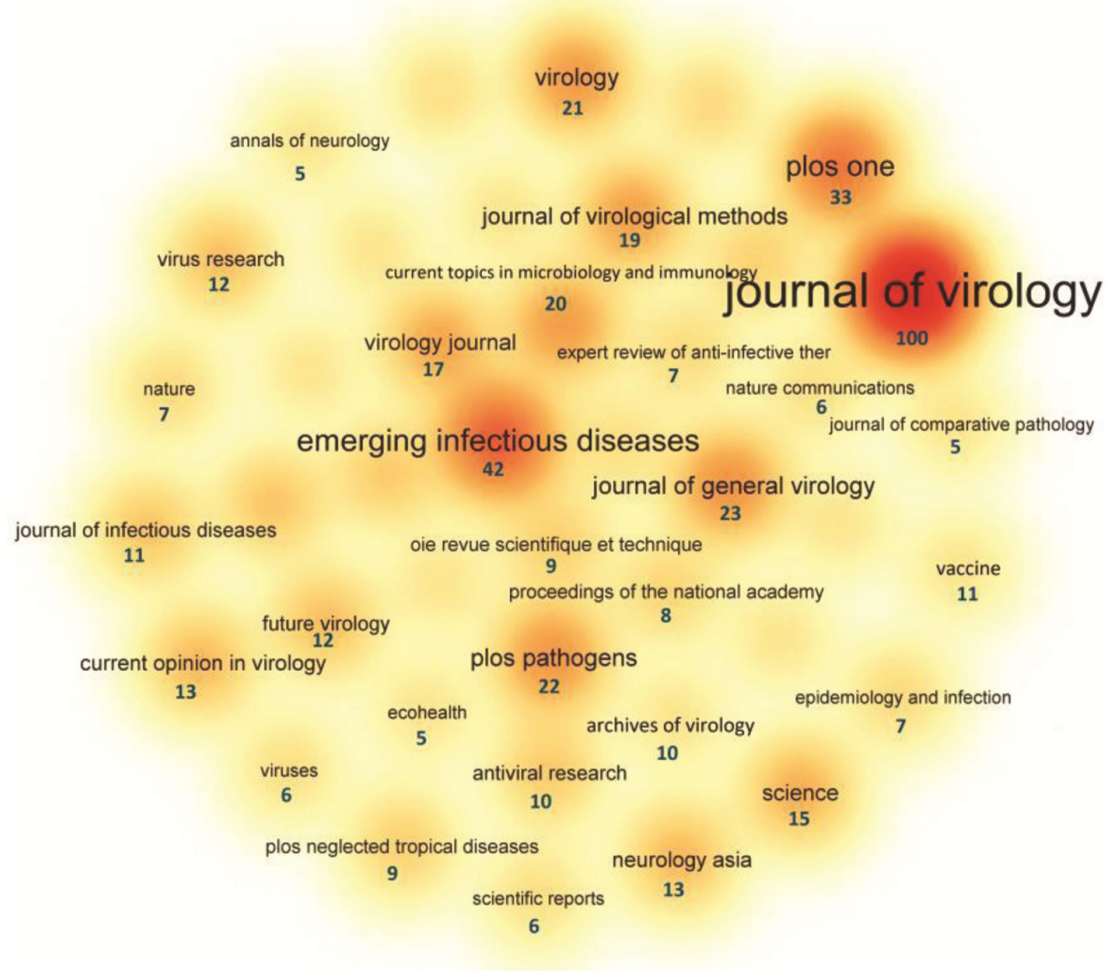

Figure 6. Journals publishing articles on Nipah virus.

Cluster 3: Centers for Disease Control and Prevention, USA; Emory University, Atlanta, USA; Institute of Epidemiology, Disease Control and Research; International Centre for Diarrheal Disease Research, Bangladesh; Northwestern University, USA and Stanford University, USA.

Cluster 4: Boston University, USA; Harvard University, USA; National Institute for Allergy and Infectious Disease; University of Liverpool, United Kingdom; University of Qeensland, Australia and University of Texas, USA.

Cluster 5: Consortium for Conservation Medicine, New York, USA; Ecohealth Alliance, New York, USA; Monash University, Malaysia; Universiti Putra Malaysia, Serdang, Malaysia and Veterinary Research Institute, Ipoh, Malaysia.

Cluster 6: Icahn School of Medicine at Mount Sinai, New York, USA; University of California, USA; University of Oxford, United Kingdom and Washington State University, USA.

Cluster 7: National Centre for Foreign Animal Diseases, Winnipeg; Philipps University of Marburg, Germany and University of Manitoba, Winnipeg, Canada

\section{Journals publishing articles on Nipah virus}

The 1007 records on Nipah virus had been published in 373 journals. The ten core journals published $31 \%$ of the total journal records. The top 40 journals publishing five or more records per journal on Nipah virus $(54 \%$ of records) are shown in Figure 6 along with the number of records published in them. Remaining 46\% (467/1007) records were published in 333 journals (1-4 records/ journal).

In Figure 6, each node represents a journal. The size and colour of nodes represent the number of records published in the respective journals. The numbers of records is also given below each node.

The Journal of Virology by the American Society for Virology has published 100 records on Nipah virus. Emerging Infectious Diseases by Centers for Disease Control (CDC) has published 42 records, the second highest number of records on Nipah virus in a journal. This is followed by PLOS ONE (USA) and Journal of General Virology (United Kingdom).

\section{Subject analysis based on keywords}

The top 62 keywords (out of total 2386) having occurred in 10 or more articles were considered for cluster analysis (Figure 7). A total of five clusters were identified by clustering schema of VOSviewer software. The nodes in Figure 7 represent keywords/subject terms and edges reveal the relation between different keywords representing subjects.

In Figure 7, O represents occurrences; L, links and LS, total link strength. The top 10 keywords along with occurrences, links and total link strength are: Hendra virus: 


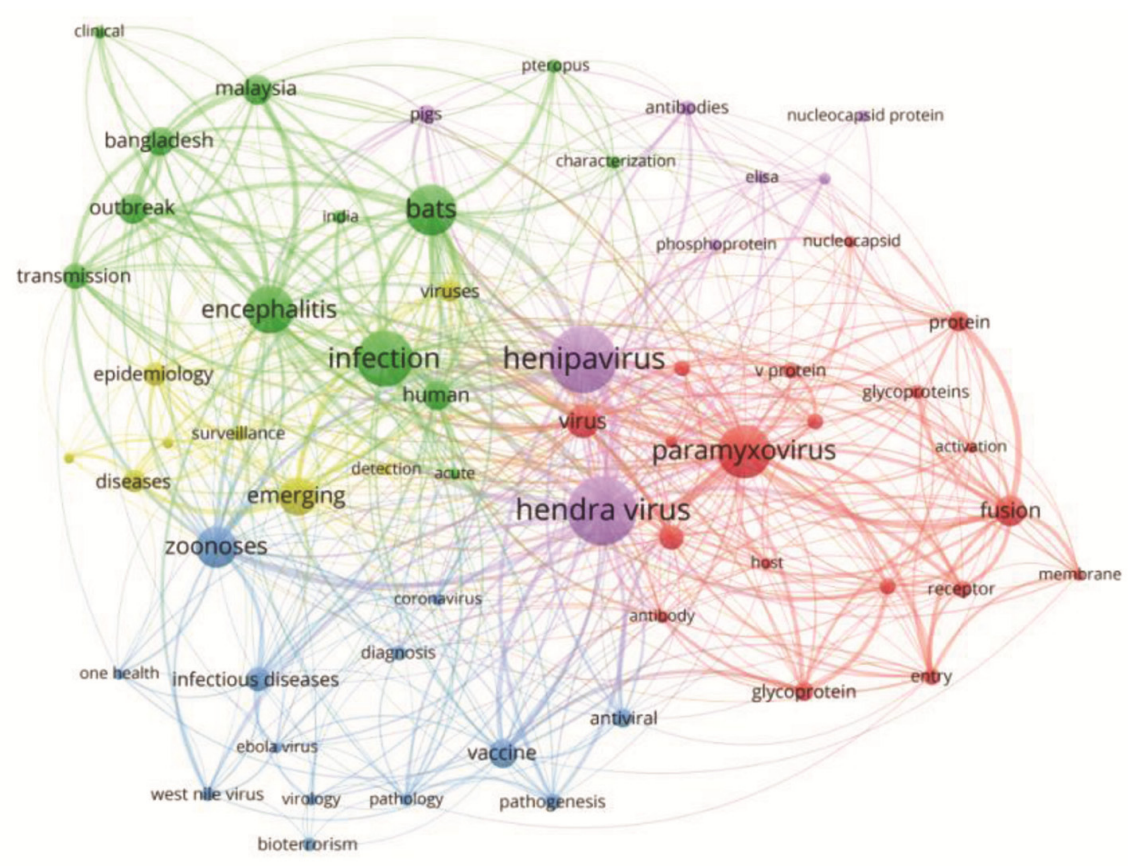

Figure 7. Keywords visualization map.

O-125, L-47, LS-210; Henipavirus: O-120, L-49, LS-188; Infection: D-96, L-43, LS-145; Paramyxovirus: D-87, L-48, LS-152; Bats: D-83, L-32, LS-147; Encephalitis: D-75, L-29, LS-124; Zoonoses: D-62, L-33, LS-118; Emerging: D-56, L-27, LS-92; Virus: D-45, L-33, LS-65; Fusion: D-42, L-20, LS-94.

Cluster 1: Activation, antibody, attachment, entry, fusion, glycoproteins, host, identification, interferon, membrane, novel, nucleocapsid, paramyxovirus, protein, receptor, v protein, viral and virus.

Cluster 2: Acute, Bangladesh, bats, characterization, clinical, encephalitis, human, India, infection, Malaysia, outbreak, pteropus and transmission.

Cluster 3: Antiviral, bioterrorism, coronavirus, diagnosis, ebola virus, infectious diseases, one health, pathogenesis, pathology, vaccine, virology, west nile virus and zoonoses.

Cluster 4: Detection, diseases, emergence, emerging, epidemiology, infectious, surveillance and viruses.

Cluster 5: Antibodies, elisa, hendra virus, henipavirus, nucleocapsid protein, paramyxoviridae, phosphoprotein and pigs.

Cluster 1 consists of 19 keywords. This shows that the characterization of Nipah virus and topics on pathogenesis have been explored in articles. Cluster 2 has 13 terms. It represents topics of clinical symptoms of Nipah virus, its transmission and locations of outbreaks. Cluster 3 also has 13 keywords. This cluster deals with the topics of transmission of infection between animals and humans, pathogeneses and vaccine development for cure of infection. Keywords in cluster 4 represent the topics of emergence of Nipah virus and its surveillance. Cluster 5 is again about characterization of Nipah virus.

\section{Major institutions and their article publishing trends in journals}

Figure 8 illustrates the top publishing institutions and journals, and how they associate in publishing trends.

The top contributor of articles, the Australian Animal Health Laboratory-CSIRO, Geelong, Australia has published articles mainly in Journal of Virology, Journal of Virological Mathods, PLoS ONE, Journal of General Virology and Virology Journal. CDC, USA published articles mainly in Emerging Infectious Diseases, the journal being published by CDC itself. In addition, articles published by CDC are included in many other journals as well. The Journal of Virology is the preferred journal for publishing articles by the Icahn School of Medicine at Mount Sinai, USA, University of California, University of Texas, Washington State University, USA, and Phillipps University, Germany. PLoS Pathogens is the preferred journal for University of California and National Institute of Allergy and Infectious Diseases, USA. After $\mathrm{CDC}$, the Emerging Infectious Diseases Journal is preferred among International Centre for Diarrheal Disease Research, Bangladesh and Ecohealth Alliance, New York, USA. The University of Malaya, Malaysia has published majority of articles in Neurology Asia (published from Malaysia) and Journal of Infectious Diseases and Emerging Infectious Diseases.

\section{Discussion}

The present study presents scientometric analysis and network visualization based on 1007 records indexed in 


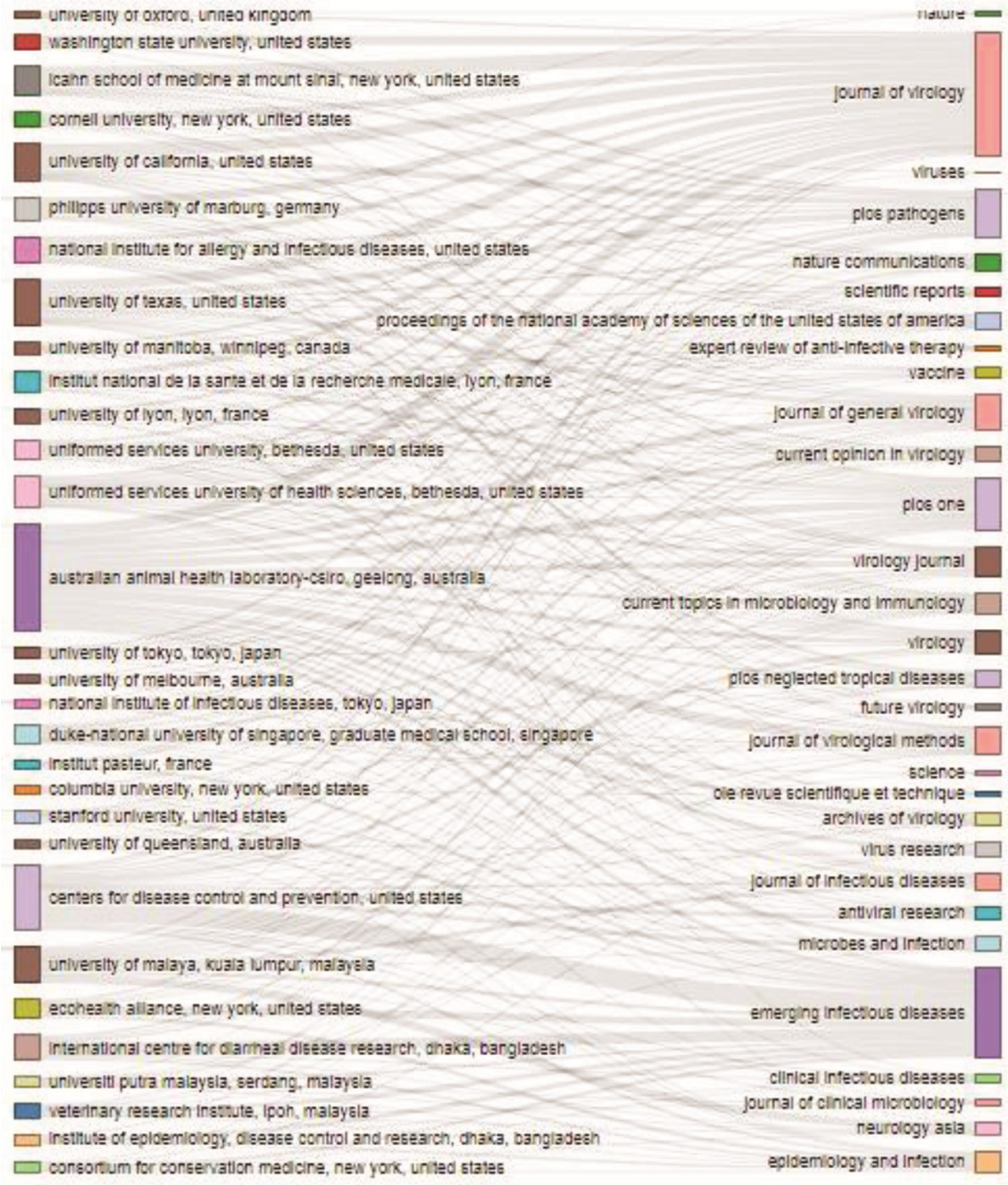

Figure 8. Institutional-journal publishing trend.

Scopus database. The analysis of chronological growth of scientific outcome on Nipah virus revealed that though $\mathrm{NiV}$ was identified two decades ago, the growth of publications on the subject is uneven. The scientific productivity on life sciences subjects is continuously increasing for the last quarter of the 20th century, whereas the growth trajectory of publications on Nipah virus appears to plateau (an average of 61.3 records between 2004 and 2017) after the first five years of discovery in 1999. Incidences of Nipah virus have been observed in Asia, viz. outbreaks have incurred in pigs in Malaysia and Singapore (1998-99), and human disease incidences have taken place in Malaysia (1998-99), Singapore (1999), Bangladesh (2001, 2003-05, 2007-15), India (2001, 2007 and 2018) and the Philippines (2014) ${ }^{14}$. Evidence of the virus without any clinical disease has also been found in fruit bats in Cambodia, Thailand and an African country, Madagascar ${ }^{10}$. The growth of articles on Nipah virus in various years remained unaffected by incidences of the disease in the respective years. It was during February 2018 that WHO included the Nipah and henipaviral diseases under the 'list of blueprint priority diseases', requiring an urgent need for accelerated research and development ${ }^{2}$. Hence, the scientific activities are expected to get an impetus in the coming years.

The analysis brought forward that there are only few productive authors on the subject. Out of total 2804 authors, only $6.3 \%$ (178) contributed to five or more articles, whereas the remaining majority of authors contributed on Nipah virus occasionally. L. F. Wang (75 documents, 155 link strength), C. C. Broder (60 documents, 125 link strength) and B. Lee (40 documents, 43 link strength) are the most productive authors on Nipah virus. Wang is presently professor and Director, Emerging Infectious Diseases Programme at Duke-NUS Medical School, Singapore. He also served at the Australian Animal Health Laboratory, Geelong, Australia. His research areas include bat-borne viruses and understanding 
virus-bat interactions. He led an international team that conducted comparative genomic analysis of two bat species and discovered links between the bat's ability to counter DNA damage repair and to co-exist with a large number of viruses without developing clinical diseases (https://www.duke-nus.edu.sg/content/wang-linfa). Broder is Professor and Director, Emerging Infectious Diseases Graduate Program, Uniformed Services University of Health Sciences, Bethesda, USA. His areas of interest are virology, emerging viruses, virus-host cell interactions, vaccines and therapeutics and viral serological surveillance. Lee works at the Department of Microbiology, Icahn School of Medicine at Mt. Sinai, New York, USA. His research topics include AIDS/HIV, antivirals, biodefence, dendritic cells, drug design and discovery, gene therapy, Nipah virus and vaccine development. Two other authors, S. P. Luby from Stanford University, USA and E. S. Gurley from International Centre for Diarrheal Diseases Research, Bangladesh, Dhaka, Bangladesh have link strength of $>100$. The contribution of 816 records $(81 \%)$ in collaborative authorship reveals the joint efforts being made to handle Nipah virus. Only 35 authors contributed to $\geq 15$ articles each, making it obvious that extensive scientific activities on the subject are limited to a few core researchers.

According to Dutta ${ }^{15}$, the 'interest in Nipah virus has remained limited to small research communities and affected countries'. However, findings of the present study are contrary to the observation of Dutta ${ }^{15}$, as the analysis of geographical distribution of journal articles depicts that authors from all over the world representing different continents made academic/research contributions towards Nipah virus, illustrating the concerns of the whole world about the infectious disease. The United States has played a leading role by contributing the highest number of records (46.8\%, 469/1007 documents) on the subject. This is in contrast to the incidences/ outbreaks of Nipah virus in Asian countries ${ }^{10}$. The United States is followed by Australia (17.9\%, 180/1007), Malaysia $(12.4 \%, 125 / 1007)$, France $(7.6 \%, 77 / 1007)$, United Kingdom (7.1\%, 71/1007) and Germany (5.4\%, 54/1007). Each of the top six countries contributed more than $5 \%$ of total records on Nipah virus. The United States has strong collaboration strength with Australia, followed by Bangladesh and Malaysia. Malaysia, South Korea, Japan and Vietnam form a different cluster based on their mutual research collaborations. During the analysis it has been noticed that out of total 81 nations, 16 contributed to more than 10 records each and 21 countries added to 3-8 records. Remaining majority of the countries were occasional contributors of 1 to 2 records only.

Through the United States is top-most contributor on Nipah virus, the Australian Animal Health LaboratoryCSIRO, Geelong, Australia is the leading institutional contributor. CDC, USA followed by University of Malaya, Malaysia and Uniformed Services University of
Health Sciences, USA are the other major contributors. CDC, USA has worked in close association with the International Centre for Diarrhoeal Disease and Research, Dhaka, Bangladesh. Besides this, CDC has also worked in strong collaboration with the Institute of Epidemiology, Disease Control and Research, Bangladesh and the Stanford University, USA. All these institutions fall in one cluster. On the other hand, the Australian Animal Health Laboratory-CSIRO, Geelong, Australia has the strongest institutional collaboration with Uniformed Services University for Health Sciences, USA. Seven of the leading 10 institutions publishing articles on Nipah virus were from the USA.

The Journal of Virology, Emerging Infectious Diseases, PLoS ONE and General Virology have emerged to be core journals publishing maximum number of articles on Nipah virus. The characterization of Nipah virus, pathogenesis, clinical symptoms, transmission and locations of outbreaks, transmission of infection between animals and humans, vaccine development and surveillance of $\mathrm{NiV}$ are the major subjects dealt in publications on Nipah virus.

The authorship, institutional and geographical analyses make it obvious that collaborative efforts are being made by scientists and institutions representing different corners of the globe to confront the challenge of Nipah virus.

\section{Conclusion}

The findings of the present study reveal that although Nipah virus was isolated two decades ago, scientific productivity on it has not yet attained the desired impetus. Only $6.3 \%$ authors (178 out of 2804 authors) contributed to more than five articles each on $\mathrm{NiV}$, making it obvious that a large majority of authors are occasional contributors on the subject. However, a great degree of authorship collaboration was observed. The initiatives of WHO to curb NiV infection are likely to boost the scientific development on zoonotic infection. Though incidences of NiV human infection have been reported in the Asian continent only, countries from all continents have been concerned about the virus. A great degree of intercountry and inter-continent collaboration was detected among United States, Australia, Malaysia, United Kingdom and Bangladesh, depicting that the developed world is working in association with the developing nations to tackle the problem. The Australian Animal Health Laboratory-CSIRO, Geelong, Australia; CDC, USA; University of Malaya, Malaysia and Uniformed Services University of Health Sciences, USA were found to be the major contributor institutions. Majority of the institutions leading the R\&D on NiV represent the USA. The scientific literature on Nipah virus is published across 373 journals, but 10 core journals published more than $30 \%$ of the articles on the subject. The characterization of Nipah virus, 
pathogenesis, clinical symptoms of the viral infection, virus transmission, outbreaks, transmission of infection between animals and humans, vaccine development, emergence of Nipah virus and its surveillance were found to be the main sub-subjects of articles. The network visualization depicting collaboration trends among major authors, countries and institutions is expected to be useful to the scientific community in analysing the research trends on Nipah virus. The findings of this study will also be useful to the institutions and policy makers to prepare the course of action to fill the research gaps.

1. Ang, B. S. P., Lim, T. C. C. and Wangf, L., Nipah virus infection. J. Clin. Microbiol., 2018, 56(6), 1-10; https://jcm.asm.org/ content/jcm/56/6/e01875-17.full.pdf

2. WHO, List of blueprint priority diseases. World Health Organization, Geneva, 2018; http://www.who.int/blueprint/priority-diseases/en/

3. Chua, K. B. et al., Fatal encephalitis due to Nipah virus among pig-farmers in Malaysia. Lancet, 1999, 354(9186), 1257-1259.

4. Chua, K. B., The discovery of Nipah virus: a personal account. Neurol. Asia, 2004, 9, 59-63.

5. Ahmad, B. and Tan, C. T., Nipah encephalitis - an update sherrini. Med. J. Malays., 2014, 69 (Suppl. A), 103-111.

6. Looi, L.-M. and Chua, K.-B., Lessons from the Nipah virus outbreak in Malaysia. Malays. J. Pathol., 2007, 29(2), 63-67.

7. OIE, World Organization for Animal Health, Nipah virus, Paris, France, 2018; http://www.oie.int/en/animal-health-in-the-world/ animal-diseases/Nipah-Virus/
8. Vandali, V. and Biradar, R. B., Nipah virus (Niv) infection: a systematic review. JOJ Nurse Health Care, 2018, 8(1), 1.

9. WHO, Nipah virus. World Health Organization, Geneva, Switzerland, 2018; https://www.who.int/news-room/fact-sheets/detail/nipahvirus

10. World Organisation for Animal Health. Nipah virus, Switzerland, 2018; http://www.oie.int/en/animal-health-in-the-world/animaldiseases/Nipah-Virus/

11. Safahieh, H., Sanni, S. A. and Zainab, A. N., International contribution to Nipah virus research 1999-2010. Malays. J. Lib. Inform. Sci., 2012, 17(3), 35-47.

12. Gupta, B. M., Ahmed, K. K. M. and Gupta, R., Nipah virus research: a scientometric assessment of global publications output during 1999-2018. Int. J. Med. Pub. Health, 2018, 8(2), 48-55.

13. Chaman Sab, M., Dharani Kumar, P. and Biradar, B. S., Scientometric study of the research performance on fishery: the Indian perspective. J. Adv. Lib. Inform. Sci., 2016, 5(2), 337-341.

14. Sharma, V., Kaushik, S., Kumar, R., Yadav, J. P. and Kaushik, S., Emerging trends of Nipah virus: a review. Rev. Med. Virol., 2019, 9(1); https://onlinelibrary.wiley.com/doi/epdf/10.1002/rmv.2010

15. Dutta, D., Nipah virus control needs more than R\&D (editorial). Lancet, 2018, 391(10137), 2295; https://www.thelancet.com/ journals/lancet/article/PIIS0140-6736(18) 312649/fulltext\#articleInformation

Received 26 June 2019; revised accepted 31 July 2019

doi: $10.18520 / \mathrm{cs} / \mathrm{v} 117 / \mathrm{i} 10 / 1574-1584$ 